\title{
Multiwall Carbon Nanotubes as Quantum Dots
}

\author{
M. R. Buitelaar, A. Bachtold,* T. Nussbaumer, M. Iqbal, and C. Schönenberger ${ }^{\dagger}$ \\ Institut für Physik, Universität Basel, Klingelbergstrasse 82, CH-4056 Basel, Switzerland
}

(Received 14 August 2001; published 28 March 2002)

\begin{abstract}
We have measured the differential conductance of individual multiwall carbon nanotubes. Coulomb blockade and energy level quantization are observed. The electron levels are nearly fourfold degenerate (including spin) and their evolution in magnetic field (Zeeman splitting) agrees with a $g$ factor of 2 . In zero magnetic field the sequential filling of states evolves with spin $S$ according to $S=0 \rightarrow 1 / 2 \rightarrow$ $0 \ldots$ A Kondo enhancement of the conductance is observed when the number of electrons on the tube is odd.
\end{abstract}

DOI: $10.1103 /$ PhysRevLett.88.156801

Carbon nanotubes (NT) are excellent model systems to study the electronic properties of low-dimensional conductors [1]. Two different classes of tubes exist: small diameter $(\sim 1 \mathrm{~nm})$ single-wall NTs (SWNT) and large diameter $(\sim 10 \mathrm{~nm})$ multiwall NTs (MWNT). Transport and scanning probe experiments on MWNTs have shown diffusive [2-4] or at best quasiballistic behavior [5] with mean free paths $l \lesssim 100 \mathrm{~nm}$. Metallic SWNTs, on the other hand, can be ballistic conductors over micron lengths [4]. Of importance in a transport experiment is the coupling of the tubes to metallic leads. Whereas transparent contacts have been standard for MWNT devices, this is a recent development for SWNTs. Most transport experiments on SWNTs have shown Coulomb blockade and a quantization of the electron states, implying that a NT quantum dot has been formed [6,7]. Only recently, transparent contacts to SWNTs could be realized. The physics of these systems proved to be very rich and ranges from devices dominated by higher-order tunneling processes (like, e.g., the Kondo effect) at intermediate contact transparencies [8] to open ballistic systems with transparencies approaching unity [9].

While there are many examples of SWNT quantum dots, little effort has gone into the investigation of MWNTs as such systems. In this Letter we demonstrate that MWNTs can form clean quantum dots as well, a nontrivial result given the larger diameter, the correspondingly smaller energy scale (subband spacing), and, most notably, the diffusive nature of the tubes. Moreover, in MWNT quantum dots the single-particle level spacing $\delta E$ can exceed the charging energy $U_{C}$, as the latter is small compared to SWNT devices. This implies that even for a lifetime broadening $\Gamma \sim U_{C}$ transport occurs via one individual quantum state. Interestingly, the electron levels are found to be nearly fourfold degenerate, a property directly related to the unique band structure of the nanotubes.

We have studied several MWNT samples with characteristics ranging from devices with very open contacts to semi-isolated quantum dots. As a reference, we will first discuss an open device. Figure 1a shows a grey-scale representation of the differential conductance as a function of source-drain $\left(V_{\mathrm{sd}}\right)$ and gate voltage $\left(V_{\mathrm{g}}\right)$ for a
PACS numbers: 73.61.Wp, 72.15.Qm, 73.21.La, 73.63.-b

$2.2 \mu \mathrm{m}$ MWNT with short $(L=300 \mathrm{~nm})$ contact spacing. A small magnetic field of $B=150 \mathrm{mT}$ is applied perpendicular to the tube axis. The linear-response conductance $G$ and the corresponding root-mean square fluctuations $\delta G_{\text {rms }}$ versus temperature are shown in Figs. 1b and 1c, respectively. Details on sample fabrication can be found in Ref. [5]. Large and reproducible fluctuations of order $e^{2} / h$ develop in $G$ versus $V_{\mathrm{g}}$. Note that the average conductance is quite large, i.e., $\langle G\rangle \sim 4.0 e^{2} / h$ and temperature independent. The conductance variation is interpreted to result from quantum interference. It differs from the patterns observed in NT samples with tunnel contacts, exhibiting Coulomb blockade (CB). In the grey-scale representation of Fig. 1a an alternating sequence of high- and low-conductance lines is seen. This pattern is not periodic. Most importantly, extended low-conductance regions bounded by high-conductance lines, as expected for $\mathrm{CB}$, are not apparent in the vicinity of $V_{\mathrm{sd}}=0 \mathrm{~V}$. Instead, well defined conductance dips develop (arrows in Fig. 1b). The irregularity of the resonances suggests the presence of scatterers along the tube and should be contrasted to the observation of periodic interference patterns in some

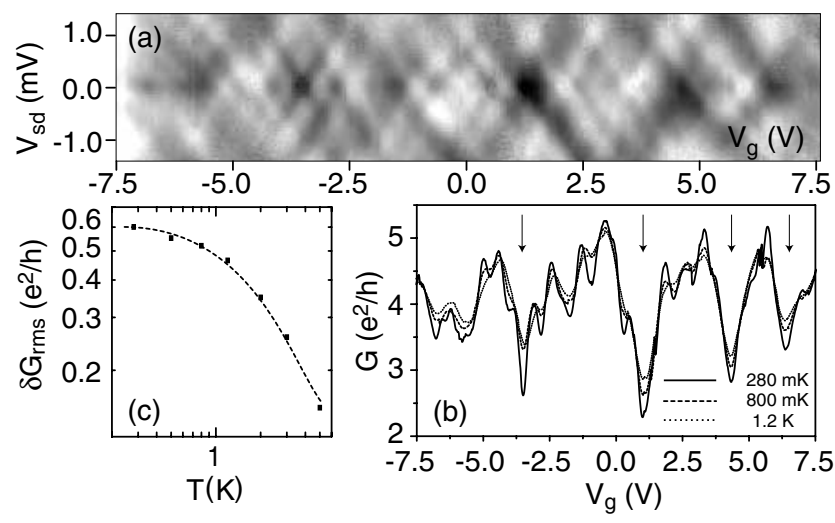

FIG. 1. (a) Grey-scale representation of the differential conductance as a function of source-drain $\left(V_{\mathrm{sd}}\right)$ and gate voltage $\left(V_{\mathrm{g}}\right)$ at $280 \mathrm{mK}$ for an open MWNT device (lighter $=$ more conductive). (b) Linear-response conductance $G$ as a function of $V_{\mathrm{g}}$. (c) The corresponding root-mean square $\delta G_{\mathrm{rms}}$ of the conductance fluctuations versus temperature. 
ballistic SWNTs [9]. The fact that $G$ often exceeds $4 e^{2} / h$ would be surprising for a SWNT but is consistent with the findings that MWNTs are substantially hole doped by the environment, allowing more than two spin-degenerate modes to contribute to the current [10].

An important length scale in the context of quantum interference is the phase-coherence length $l_{\phi}$. Information about $l_{\phi}$ can be obtained from the root-mean square of the conductance fluctuations; see Fig. 1c. For disordered wires random variations of $G$ as a function of magnetic field or Fermi energy (which is changed here with the gate) are usually assigned to universal conductance fluctuations (UCF). These fluctuations depend on the specific scattering potential but are universal in the sense that their amplitude at zero temperature is of the order $e^{2} / h$ regardless of the sample size and degree of disorder [11]. For a wire that is $1 \mathrm{D}$ with respect to $l_{\phi}$ one expects a crossover to the universal value $\delta G_{\mathrm{rms}}=0.73 e^{2} / h$ when $l_{\phi} \sim L$. This appears in the measurement at $T \lesssim 1 \mathrm{~K}$ and $\delta G_{\text {rms }}$ saturates around $0.6 e^{2} / h$ at $T_{0}=280 \mathrm{mK}$, in good agreement with theory. Note that the applied field of $150 \mathrm{mT}$ is not enough to fully destroy time-reversal symmetry, in which case $0.52 e^{2} / h$ is expected. The saturation of $\delta G_{\mathrm{rms}}$ close to the universal limit suggests conduction through one phase coherent unit. This in turn implies that $l_{\phi} \gtrsim L$ at $T_{0}$.

Figure 2 shows a grey-scale plot of the differential conductance of another $2.3 \mu \mathrm{m}$ MWNT device (electrode spacing $L=300 \mathrm{~nm}$ ) as a function of $V_{\mathrm{g}}$ and $V_{\mathrm{sd}}$ for relatively large positive gate voltages. In the range $V_{\mathrm{g}} \lesssim 10 \mathrm{~V}$ (not shown) the data resemble Fig. 1. In contrast to the first device, however, clear and regular signatures of $\mathrm{CB}$ are visible when $V_{\mathrm{g}}$ is increased beyond $V_{\mathrm{g}} \sim 12 \mathrm{~V}$. These changes are accompanied by a gradually decreasing $\langle G\rangle$ as $V_{\mathrm{g}}$ is increased. The most striking observation in Fig. 2 is a sequence of a large low-conduction "diamond" followed by three smaller ones (best seen on the left). The diamonds are highlighted by dashed lines in the figure. The size of the diamonds reflects the magnitude of the addition energy $\Delta E_{\text {add }}$, which measures the difference in chemical potential of two adjacent charge states of the dot. In the constant interaction model $\Delta E_{\text {add }}=U_{C}+\delta E$, where $U_{C}=e^{2} / C$ is the single-electron charging energy and $C$ the electrostatic capacitance [12]. If all the single-electron levels would repel each other (only twofold spin degeneracy) and $\delta E \sim U_{C}$, an alternating sequence of small and large diamonds would be expected. Starting from an even filling number, $\Delta E_{\text {add }}=U_{C}+\delta E$ for the first added electron (large diamond) and $U_{C}$ for the second one (small diamond) [13]. The sequence of one large diamond followed by three smaller ones of approximately equal size, which is observed here, suggests that the degeneracy of the states is not 2, but rather 4 (including spin). From the size of the diamonds we obtain $\delta E \approx 0.8 \mathrm{meV}$ and $U_{C} \approx 0.4 \mathrm{meV}$, the latter corresponding to $C_{\Sigma}=400 \mathrm{aF}$. The total capacitance $C_{\Sigma}$ is the sum of the gate capacitance $C_{\mathrm{g}}$ and the contact capacitances $C_{\mathrm{s}}$ (source) and $C_{\mathrm{d}}$

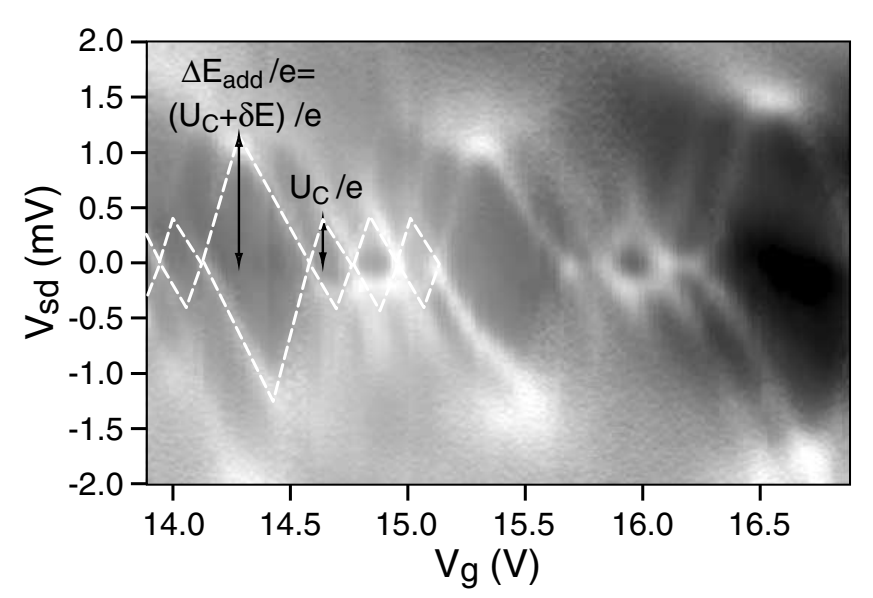

FIG. 2. Grey-scale representation of the differential conductance as a function of source-drain $\left(V_{\mathrm{sd}}\right)$ and gate voltage $\left(V_{\mathrm{g}}\right)$ at $280 \mathrm{mK}$ (lighter $=$ more conductive). The average twoterminal conductance is high $\left(\sim 2 e^{2} / h\right)$; nevertheless, clear traces of Coulomb blockade are observed. The pattern of a large diamond followed by three smaller ones suggests a (nearly) fourfold degeneracy (including spin) of the single-electron dot states. $\Delta E_{\text {add }}, U_{C}$, and $\delta E$ denote the addition energy, the charging energy, and the single-electron level spacing, respectively.

(drain). All three parameters can be deduced from the diamonds. We obtain $C_{\mathrm{g}}=1 \mathrm{aF}$ and $C_{\mathrm{s}, \mathrm{d}}=260,140 \mathrm{aF}$. An estimate of the lifetime broadening $\Gamma$ can be obtained from the measured width of the Coulomb peaks and yields $\Gamma \approx 0.25 \mathrm{meV}$.

The appearance of regularly spaced electron states seems puzzling given the disorder and the substantial hole doping in MWNTs. To reconcile this we propose transport through a semiconducting outermost shell and a metallic second inner one for this device. The semiconducting shell is hole doped by the environment [10] and conducts at moderate $V_{\mathrm{g}}$. At large positive gate voltages, however, this tube will be depleted of charge carriers and the second shell (assumed to be metallic) will dominate electron transport, as observed in Ref. [14]. Separated from the dopants on the outside of the MWNT, the inner shell then constitutes a clean and largely undoped quantum dot. As the coupling to the leads decays exponentially for inner shells [14], we do not expect more shells to contribute to the current at low temperatures. For the same reason we expect this quantum dot to be extended over the whole NT length.

The level spacing $\delta E$ of an ideal and undoped metallic NT is given by $\delta E=h v_{\mathrm{F}} / 2 L$, where $v_{\mathrm{F}}=8 \times 10^{5} \mathrm{~m} / \mathrm{s}$ is the Fermi velocity [15]. Assuming $L$ to be the complete tube length of $2.3 \mu \mathrm{m}$, this yields $\delta E=0.72 \mathrm{meV}$ in good agreement with the observed $\delta E \approx 0.8 \mathrm{meV}$. Transport through a clean and undoped tube would also explain why the large diamonds of Fig. 2 look uniform. This would not be the case if more than the ideally expected two modes participate in transport. The appearance of quantized states also shows that transport can be phase 
coherent over distances of order $1 \mu \mathrm{m}$, in agreement with the findings for the more open device.

The observation of a ratio $\delta E / U_{C}>1$ is particularly interesting. The level spacing $\delta E$ of an undoped tube is independent of diameter and depends only on $L$. The charging energy, on the other hand, is found to be dominated by the contact capacitances. Given the 10 times larger diameter and overlap with electrodes, MWNTs are more likely to have a small $U_{C}$ as compared to SWNTs, yielding large $\delta E / U_{C}$ ratios.

The observed fourfold degeneracy can be explained by a specific property of the graphite sheet (graphene). In the simplest tight-binding band-structure calculation all 1D bands are twofold degenerate (not including spin) [15]. This degeneracy can be traced back to the presence of two $\mathrm{C}$ atoms per unit cell, each contributing with one valence orbital. This so-called $K-K^{\prime}$ degeneracy has not been observed before, although it is supposed to be a generic feature of graphene.

To explore this scenario further we have also studied the gate-voltage shifts of the linear-response conductance peaks as a function of a magnetic field $B$ perpendicular to the tube axis, Fig. 3. The difference between the positions of adjacent peaks can be related to the addition energy: $e \Delta V_{\mathrm{g}}=\Delta E_{\mathrm{add}} C_{\Sigma} / C_{\mathrm{g}}$. Figure 3a shows the evolution in small magnetic field $B \leq 3 \mathrm{~T}$. Adjacent peaks are seen to shift in opposite directions. This is the behavior of a ground state whose spin alternates as $S=0 \rightarrow 1 / 2 \rightarrow$ $0 \ldots$ This indicates that the fourfold degeneracy is not exact as we will explain now: In the presence of a magnetic field the energy of an electron depends on its spin due to the Zeeman effect which lowers the degeneracy from 4 to 2 . If such a quartet is half-filled with $N=2$ electrons, it is energetically more favorable for the two electrons to occupy different orbitals with parallel spin. This spin alignment is actually already expected for $B=0$, because of exchange correlations. Hund's rule would favor the spin triplet with total angular momentum $S=1$ when the gain in exchange energy $E_{X}$ exceeds the level spacing between single-particle states [16]. The spin should therefore evolve as $S=1 / 2 \rightarrow 1$ for $N=1 \rightarrow 2$. Experimentally, however, the first two electrons have opposite spins and are thus added to the same orbital state. This discrepancy can be resolved only if the assumed fourfold degeneracy is relaxed, i.e., there are pairs of states which lie close together with spacing $\delta E^{\star}$. The pairs themselves are spaced by $\delta E \gg \delta E^{\star}$. A detailed study of the peak evolutions (Fig. 3b) reveals that this is indeed the case. $\Delta E_{\text {add }}$ at $B=0$ of the $2 \rightarrow 3(6 \rightarrow 7)$ transition is clearly larger than the $1 \rightarrow 2$ and $3 \rightarrow 4(5 \rightarrow 6$ and $7 \rightarrow 8)$ ones. We obtain $\delta E_{23}^{\star} \approx 0.1 \mathrm{meV}$ and $\delta E_{67}^{\star} \approx 0.18 \mathrm{meV}$. We have also verified that the energy shifts agree with the Zeeman term for electrons occupying the same orbital. We plot in Fig. $3 \mathrm{c}$ the corresponding addition energies as a function of $B$. A best fit of the data to $U_{C}+g \mu_{B} B$, where $\mu_{B}$ is the Bohr magneton and $g$ the Landé factor, is shown as a dashed line and yields $g=2.1 \pm 0.3$. This value
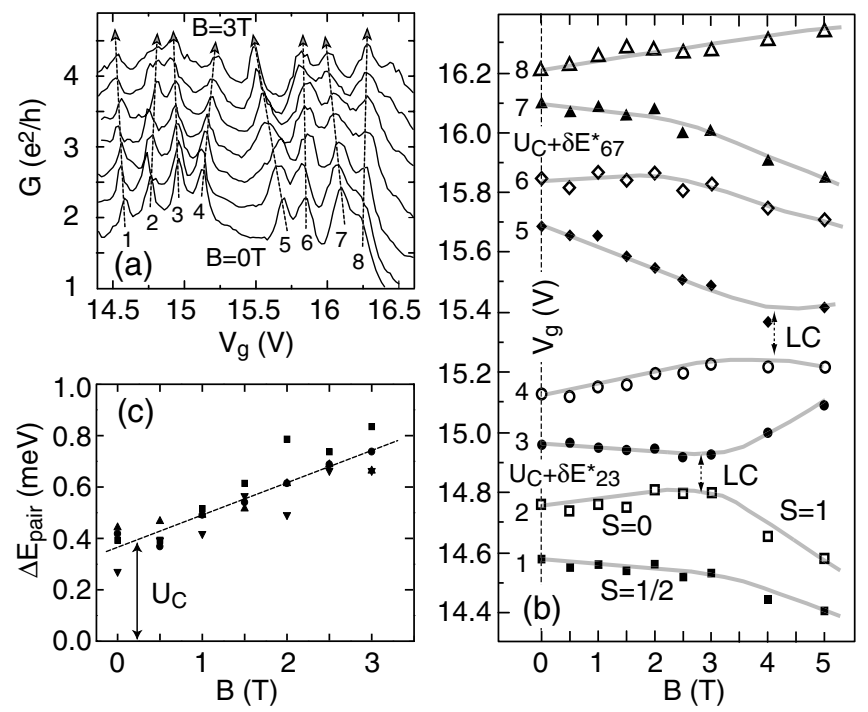

FIG. 3. (a) Linear-response conductance $G$ as a function of gate voltage $V_{\mathrm{g}}$ for different magnetic fields $B=0, \ldots, 3 \mathrm{~T}$ (vertically offset for clarity). The evolution of the conductance peaks are highlighted by dashed lines. (b) Peak positions versus $B=0, \ldots, 5 \mathrm{~T}$. Curves are guides to the eye and LC denotes level crossings. (c) Magnetic field dependence of the addition energy deduced from the separation of adjacent peaks involving electrons on the same orbital (the pairs $1 \leftrightarrow 2,3 \leftrightarrow 4,5 \leftrightarrow 6$, and $7 \leftrightarrow 8$ ). The dashed line (fit) corresponds to the Zeeman energy with Landé factor $g=2.1$.

is consistent with $g=2.0$ for graphite and with previous measurements of $g$ for a SWNT $[6,17]$.

In high magnetic field levels cross. Two level crossings (LCs) are seen in Fig. 3b. At $B \approx 3 \mathrm{~T}$, for example, the spin up of the first orbital crosses the spin down of the second, giving rise to an $S=0 \rightarrow 1$ transition. A similar crossing is not seen in the upper part. On the one hand, this is due to the larger $\delta E^{\star}$. On the other hand, there is also a magnetic-field dependence of the orbitals which increases $\delta E^{\star}$ at higher fields.

We find a pattern that repeats every fourth electron due to an apparent pairing of orbital states. We believe that this pairing is related to the $K-K^{\prime}$ degeneracy. Hybridization via the contacts is proposed as a possible mechanism for the slight level splitting $\delta E^{\star}$ of the orbital states, which in size is comparable to the lifetime broadening. Alternatively, scatterers which lower the symmetry of the system may cause the level splitting. In high magnetic field the intrinsic $K-K^{\prime}$ degeneracy should be lifted, which enhances the level separation further. This may explain why the $S=0 \rightarrow 1$ transition is not observed for the upper quartet in Fig. 3b. Finally, the fact that $S=0$ for $N=2$ at $B=0$ is consistent with Hund's rule only if the exchange energy $E_{X}<\delta E^{\star}$, yielding an upper bound for $E_{X}$ of $\sim 0.1 \mathrm{meV}$.

Another interesting manifestation of the electron spin on the electric transport can be seen in the gate region between 15.5 and $16.5 \mathrm{~V}$; see Fig. 4. In the valleys marked as $E$ (even filling) the conduction decreases with decreasing temperature, while it increases in the valleys marked as 


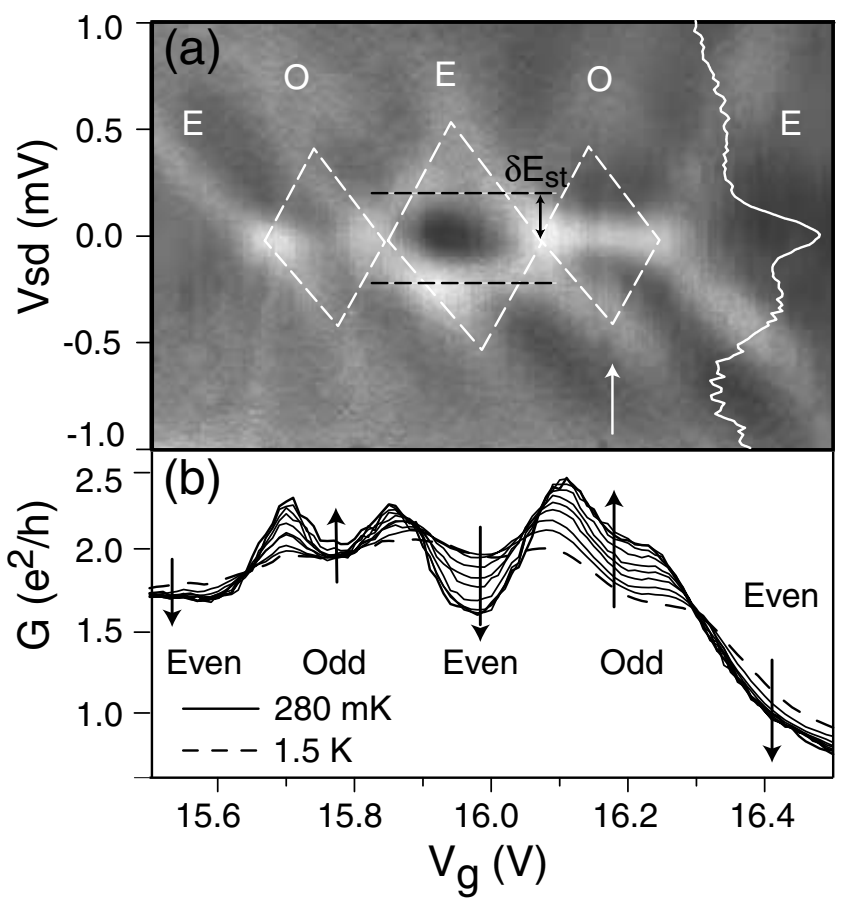

FIG. 4. (a) Grey-scale representation of $d I / d V$ as a function of source-drain $\left(V_{\mathrm{sd}}\right)$ and gate voltage $\left(V_{\mathrm{g}}\right)$ at $280 \mathrm{mK}$ (lighter $=$ more conductive). Within regions marked as $O(E)$ the number of electrons on the tube is odd (even). The horizontal features are caused by the Kondo effect. (b) Temperature dependence of the linear-response conductance. The arrows indicate directions of decreasing temperature.

$O$ (odd filling). Contrary to what one might expect from normal $\mathrm{CB}$, a high conduction "ridge" around $V_{\mathrm{sd}}=0 \mathrm{~V}$ develops in the latter. These observations can be understood with the Kondo model $[8,18]$. When the number of electrons on the tube is odd and the coupling to the leads is strong enough to allow for higher-order tunneling processes, a spin singlet can form between the spin polarized tube and electrons in the leads. This results in a resonance in the density of states at the Fermi energy (i.e., the Kondo resonance). The width of the Kondo resonance reflects the binding energy of the singlet which is usually described by a Kondo temperature $T_{K}$. The conductance is expected to increase logarithmically with decreasing temperature in the centers of the ridges below $T_{K}$. Following $G$ as a function of temperature at $V_{\mathrm{g}}=16.2 \mathrm{~V}$ we indeed find a logarithmic dependence between $280 \mathrm{mK}$ and $1 \mathrm{~K}$. At temperatures well below $T_{K}$ the conductance is expected to saturate at the unitary limit of $2 e^{2} / h$. In our case, however, no saturation has been observed down to $280 \mathrm{mK}$.

The Kondo effect is expected to be suppressed by a small bias voltage across the tube of the order of $\pm k_{B} T_{K} / e$. The ridge at $V_{\mathrm{g}}=16.2 \mathrm{~V}$ has a width of $\sim 0.2 \mathrm{meV}$ which would correspond to $T_{K}=1.2 \mathrm{~K}$ (see curve in Fig. 4a). This is in agreement with the onset of the logarithmic increase of $G$ below $\sim 1 \mathrm{~K}$. An additional prediction is the disappearance of the Kondo resonance in a magnetic field. The high conductance ridge indeed broadens and disappears above $\sim 1.5 \mathrm{~T}$. Simultaneously, the Coulomb blockade diamonds are recovered. In Fig. 4a the boundary of the CB region at half-filling is clearly seen to be distorted into a truncated diamond. The associated energy $\delta E_{s t}=0.20 \pm 0.05 \mathrm{meV}$, indicated in the figure, corresponds to the energy difference between the singlet ground state and the triplet excited state $[8,19]$. The relation $\delta E_{s t}=\delta E_{67}^{\star}-E_{X}$ together with $\delta E_{67}^{\star} \sim 0.18 \mathrm{meV}$ shows that $E_{X}$ must indeed be small.

In conclusion, we show that MWNTs can form clean quantum dots in which the level separation exceeds the charging energy. Moreover, we observe a pairing of $0 \mathrm{D}$ states which we propose to be caused by the $K-K^{\prime}$ degeneracy generic to graphene. Since adsorbates on the outside of a NT are a likely source of scattering, the ability to selectively address clean inner shells of a MWNT may prove advantageous in this regard.

We acknowledge W. Belzig, G. Burkard, D. Cobden, R. Egger, and J. Nygård for discussions. We thank L. Forró for the MWNT material and J. Gobrecht for providing the oxidized Si substrates. This work has been supported by the Swiss NFS.

*Present address: École Normale Supérieure, Paris, France. $\dagger$ Electronic address: Christian.Schoenenberger@unibas.ch

[1] For reviews, see C. Dekker, Phys. Today 52, No. 5, 22-28 (1999); C. Schönenberger and L. Forró, Phys. World 13, No. 6, 37-41 (2001).

[2] L. Langer et al., Phys. Rev. Lett. 76, 479 (1996).

[3] A. Bachtold et al., Nature (London) 397, 673 (1999).

[4] A. Bachtold et al., Phys. Rev. Lett. 84, 6082 (2000).

[5] C. Schönenberger, A. Bachtold, C. Strunk, J.-P. Salvetat, and L. Forró, Appl. Phys. A 69, 283 (1999).

[6] S. J. Tans et al., Nature (London) 386, 474 (1997).

[7] M. Bockrath et al., Science 275, 1922 (1997).

[8] J. Nygård, D. H. Cobden, and P. E. Lindelof, Nature (London) 408, 342 (2000).

[9] W. Liang et al., Nature (London) 411, 665 (2001).

[10] M. Krüger, M. R. Buitelaar, T. Nussbaumer, and C. Schönenberger, Appl. Phys. Lett. 78, 1291 (2001).

[11] P. A. Lee and A. D. Stone, Phys. Rev. Lett. 55, 1622 (1985).

[12] L. P. Kouwenhoven et al., in Mesoscopic Electron Transport, edited by L. L. Sohn et al. (Kluwer, Dordrecht, The Netherlands, 1997).

[13] J. Nygård, Ph. D. thesis, University of Copenhagen, 2000.

[14] P. G. Collins, M. S. Arnold, and P. Avouris, Science 292, 706 (2001).

[15] See, e.g., M. S. Dresselhaus, G. Dresselhaus, and P. C. Eklund, Science of Fullerenes and Carbon Nanotubes (Academic Press, New York, 1996).

[16] S. Tarucha, D. G. Austing, Y. Tokura, W. G. van der Wiel, and L. P. Kouwenhoven, Phys. Rev. Lett. 84, 2485 (2000).

[17] D. H. Cobden, M. Bockrath, P. L. McEuen, A. G. Rinzler, and R. E. Smalley, Phys. Rev. Lett. 81, 681 (1998).

[18] For a review see L. Kouwenhoven and L. Glazman, Phys. World 14, No. 1, 33-38 (2001).

[19] S. De Franceschi et al., Phys. Rev. Lett. 86, 878 (2001). 